\title{
Derailment detection and data collection in freight trains, based on a wireless sensor network
}

\author{
M. Macucci*, S. Di Pascoli*, P. Marconcini*, B. Tellini ${ }^{\dagger}$ \\ *Dipartimento di Ingegneria dell'Informazione, Università di Pisa \\ Via G. Caruso 16, I-56122 Pisa, Italy \\ Email: macucci@mercurio.iet.unipi.it \\ † Dipartimento di Ingegneria dell’Energia, dei Sistemi, del Territorio e delle Costruzioni, \\ Università di Pisa, Largo Lucio Lazzarino, I-56122 Pisa, Italy
}

\begin{abstract}
We report the development of a network of wireless ultra-low-power sensors to be deployed on freight railway cars, with the main purpose of detecting derailment events and alerting the engineer in the cab of the leading locomotive. Since no power bus is available on freight cars, we plan to rely on energy scavenging from vibrations, therefore minimization of the power consumption has been one of our main priorities. We have therefore focused on ultra-low-power hardware and strived to reduce the time intervals during which it is in active mode, achieving an average power consumption below $0.5 \mathrm{~mW}$ with an active cycle of about $20 \mathrm{~ms}$ every $2 \mathrm{~s}$. We discuss the overall concept that we propose, including the self-initialization protocol and the communication strategy we have developed, and present the results of measurements on a prototype network that we have implemented.
\end{abstract}

\section{INTRODUCTION}

The derailment of a freight car may occur as the result of failure of the wheels or axles, damaged rails, poorly maintained switches, extraneous objects on the tracks, and is one of the most common railway accidents. If such a derailment initially goes unnoticed by the train crew, because of the length of the train and the high noise level, severe consequences may ensue. Indeed a derailed car can damage kilometers of track, be an obstacle for a train running in the opposite direction on the adjacent track, even lead to extremely serious events. In particular, if dangerous goods are involved, such as in the derailments of Liquid Propane Gas (LPG) tank cars, the outcome may be extensive loss of property and even of lives: Crescent City, IL was obliterated in 1970 by the explosion of derailed LPG cars, and in 2009 the ignition of the LPG that had escaped from a derailed ruptured tank car caused 32 casualties in Viareggio, Italy.

It is therefore apparent that the early detection of a derailed axle is a problem of significant importance, because, if prompt action is taken by the train driver, in about $40 \%$ of total derailments much more serious consequences can be prevented, according to the data of the European Railway Agency [1]. However it is essential to limit the occurrence of false positives and to contain the costs of the equipment to be added to freight cars, otherwise a large scale adoption is unlikely. Many efforts have been spent in designing and implementing devices that can detect the derailment condition and, in most existing solutions, initiate an emergency brake application.
The traditional approach is based on a mechanical device attached to the two ends of a car and connected with the train brake line [2]: if, as a result of derailment, the amplitude of vibrations overcomes a given threshold, air is released from the brake line, resulting in an immediate emergency stop. There are several disadvantages associated with this type of detectors, which have severely limited their diffusion. First of all, the mechanical implementation leads to costs of the order of thousands of Euros, furthermore installation is relatively complex, because the detector becomes part of the brake system of the train, and therefore needs to be carried out by specialized personnel. This has limited the application of such detectors, and a debate developed between the RID Committee of Experts (Committee for the transportation of dangerous goods), which had recommended [3] to make the installation of a mechanical derailment detector compulsory on all cars for the transportation of dangerous goods built after January 1st 2011 and the European Railway Agency, which in its final report [1] advised not to follow such a recommendation.

Among the reasons indicated by the European Railway Agency is the fact that such detectors may trigger an emergency brake application in situations in which it may be unwise to do so, and it would be better to leave to the driver the decision on how to cope with an ongoing derailment.

An approach in this direction has been proposed by A. V. Vostroukhov et al. [4], who have investigated the spectral characteristics of the vibrations under the wheels of the locomotive when a car derails. The anomalous vibrations due to the derailment are transmitted along the rails and have a small but appreciable effect under the locomotive. A refined spectral analysis is needed to extract the signature of the derailment. However the further the derailed car is from the engine, the smaller the measured effect will be, and the reliability of the method is too dependent on the, in principle widely variable, mechanical characteristics of the railbed.

Our concept, which has been recently presented [5], is an extension of what has already been done on some passenger trains: a solid-state accelerometer measures the vertical component of the acceleration, and, if a derailment condition is recognized, an alarm signal is sent to the train driver. On modern passenger trains, where a data bus and a power source are available on all cars, the implementation is rather 
straightforward and has been successfully achieved in Japan: a report by M. Kato and K. Terada [6] presents three criteria that can be applied to the accelerometer output to decide whether a derailment has occurred. Such criteria have been thoroughly tested on the Keiyo line in Japan, with successful results.

The difficulty in extending this concept to freight trains derives from the fact that on freight trains there is no availability of power or of a data communication line. We address these issues with energy scavenging from vibrations and wireless radio communication.

The power needed for the operation of the sensor node can be obtained from mechanical vibrations, which do have a significant amplitude during the normal motion of a freight car. In the literature, several approaches can be found to the conversion into electrical power of mechanical vibrations: electromagnetic (inductive) [7]-[11], electrostatic (capacitive) [12] and piezoelectric [13], [14]. All of these techniques have been used to power both sensors and actuators. A difficulty, which may affect the type of application we are interested in, in particular when the train moves at reduced speed, is represented by the fact that the maximum voltage and generated power drop dramatically at low vibration frequency. There are, however, techniques that have been proposed to overcome this problem, based on mechanically up-converting the ambient vibration frequency, as suggested by Külah and Najafi [15]. For our application the best solution appears to be that of the electromagnetic harvester, based on a magnetic mass moving within a solenoid as a result of vibrations. Perpetuum Ltd. [7]. produces electromagnetic energy scavengers that they apply to the collection of vibration and temperature data from a train axle and wireless transfer of this information to a data collection system on the train. They install the scavenger directly on the unsprung mass, where vibrations are maximum and, for their model PMG-37, obtain more than $90 \mathrm{~mW}$ of output for an RMS (Root Mean Square) acceleration of $1 \mathrm{~g}$. In our case, it is much preferable to attach the nodes to the car body, in order to make the installation quicker, less expensive, and requiring much less effort for its certification. Although on the car body the RMS amplitude of the vibration is reduced, significant power can still be obtained, as shown in Ref. [8], where $80 \mathrm{~mW}$ are shown to be achievable for a freight car traveling at $80 \mathrm{~km} / \mathrm{h}$. Energy harvesters based on the piezoelectric effect are in general cheaper and smaller, but supplying less power compared to electromagnetic units. Some piezoelectric harvester could however match the requirements for our nodes, since they can deliver an average power of the order of $10 \mathrm{~mW}[16]$.

Any energy scavenging approach must be supplemented with some form of energy storage, capable of compensating the unavoidable fluctuations in power output. Such a storage can consist in rechargeable batteries or in supercapacitors: for the specific application we are considering, supercapacitors appear as preferable, because of the short-term storage that is needed and because of the requirements in terms of reliability and lifetime. Indeed, each sensor node should have a lifetime of at least 10 years, preferably 20 years, without any maintenance. In addition a proper optimized two-stage DC-DC converter must be designed in order to make the energy harvester work in the load condition yielding the largest possible power at any train speed, and at the same time delivering a constant voltage to the load. Such a converter will also fully charge the supercapacitors (or batteries) to prepare them for the network initialization and configuration phase.

A search we have performed of existing patents has turned up an idea [17] that has some analogy with our main concept, as far as the creation of a wireless sensor network along a train is concerned, but differs under several other points of view and appears to be mainly the result of a purely theoretical study, without an effort to produce and test a prototype. The proponents of Ref. [17] have conceived a wireless network of acceleration sensors included in the bearing cover of each wheel, which have the purpose of monitoring the mechanical conditions of the bearing itself. In this case power is obtained from an embedded generator exploiting the axle rotation, and a relatively complex processing of the information from the accelerometers is needed to provide accurate data. They suggest to use information from the spectral content to determine the speed at which each car is moving, to gather information about the bearing status and to detect anomalies. Due to our requirements about the ease of installation and the low cost, we cannot follow this approach, in particular because it requires a relatively complex action on the bearing.

Furthermore, it would involve a relatively complex certification process, because modifications to mechanical parts that are critical for the safety of operation would take place. We focus instead on the development of a system characterized by an extremely simple installation procedure, that can be performed by unskilled personnel. In addition, we propose a complete system, including both the derailment sensors and a communication network covering the whole length of the train, and which can be used also for carrying additional information, such as readings of humidity/temperature sensors or of payload tags, to mention just a few examples.

There are alternative energy scavenging approaches that could be exploited, in order to increase the amount of energy available, but it is not easy to establish their long-term reliability without extensive testing. A possibility could be the exploitation of the air flow resulting from the motion of the train, with a propeller connected to a generator or with the conversion of vibrations induced by such an air flow in a properly designed cavity. An interesting solution for wireless sensors powered by air flow has been proposed by Sardini and Serpelloni [18], who have designed and implemented selfpowered wireless sensors located in a duct where an air flow is present. Although this is certainly an interesting possibility, in a railway environment there is a risk of dust and dirt accumulation, which could cause failure of the sensor power supply in a few years or even in a few months.

There are other issues that we will then need to evaluate on the field, in particular from the point of view of ElectroMagnetic Interferences (EMI) in a real-life railway environment, which may lead to loss of data. Analogous problems have been observed for wireless data communication in an industrial environment by Lee et al. [19]. Therefore we plan to collect data in a typical freight train environment, as discussed later.

The present paper is an extension of our contribution [5] to the 2015 M\&N Conference, with the addition of a more indepth and more quantitative analysis of the energy budget, of 


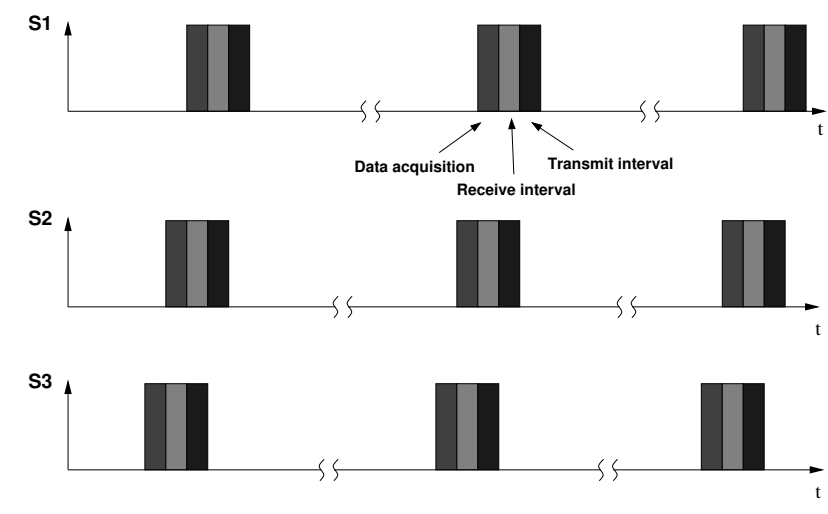

Fig. 1. Sketch of the sequential operation of the sensor nodes

the description of an improved algorithm for configuration and operation, and of experimental results obtained on a prototype network, including a test of the functionality of the radio links on a real train and a practical verification of the initial configuration algorithm.

\section{COMMUNICATION PROTOCOLS AND NETWORK CONFIGURATION}

Low power, low-cost wireless sensor networks have received a lot of attention in the recent literature [20]-[23] and find applications in many fields. The wireless network that is needed for our derailment detection system falls in the category of "ad-hoc" networks, in the sense that it is a network whose nodes and link routes are not known before the individual nodes come into life and start communicating. Many algorithms have been developed that allow the configuration of such a network, from the hypothesis of an arbitrary spatial distribution of nodes. In our case this can be significantly simplified, since all the sensor nodes are located along a train, at intervals that are approximately known. The nodes are all identical, except for the master node in the locomotive, which is powered from the locomotive itself and can also be interfaced with long-distance communication equipment.

Before discussing the initial configuration of the network, there is an important issue that we would like to present first. The main energy consumption is the result of data acquisition and processing, data reception, and data transmission. Therefore it is imperative that each of these steps is optimized in terms of duration. The patent in Ref. [17] assumes that the nodes are in a low-consumption sleep mode until they receive an interrupt that wakes them up. Such an interrupt is assumed as a result of a particular signal from the sensor or of a received signal from another node. In our opinion this approach is most likely unfeasible in practice, because the relevance of the signal from the sensor is determined on the basis of some processing of the raw data, which must be performed with the node in an active state, and, more importantly, there is no way to wake up a node with the radio signal from another node if the receiver is not in an active state, which requires a power comparable to that of the active state of the whole node. A reasonable option that we envisage, which minimizes energy consumption and which is at the same time compatible with proper operation of this type of sensor network, is based on a careful time scheduling and synchronization of the active phases of the different nodes. A possible sequence is represented in Fig. 1. Each node will wake up in the slot that it has been assigned during the configuration phase, activate the accelerometer and interpret its data. Then it will turn on the receiver and wait for the transmission from its nearest upstream (with reference to the communication flow along the train) neighbor and then operate a transmission, which, in the case that the local accelerometer data indicate no derailment, will just be a relaying of the data from the preceding node, otherwise will be a derailment alert. Periodically, also a communication in the opposite direction, from the locomotive to the tail of the train, can take place. If needed, further refinements of the network synchronization can be achieved with the techniques developed by Berger et al. [24], who have devised an ingenuous algorithm for the precise synchronization of nodes characterized by limited computing power.

In a relatively short train, up to $30-40$ cars, there will be a single node transmitting at each time, while in longer trains there could be two or more nodes transmitting at the same time, as long as they are far enough not to interfere with each other. If the nodes transmit with the highest possible power of $10 \mathrm{dBm}$, coverage will extend over a length of several cars, thereby granting proper operation even in the case of failure of one or more nodes. However, at a distance of 20 or more cars, there will be no interference if two nodes transmit at the same time, allowing multiple simultaneous flows of data, useful to reduce the interval between two consecutive data transmissions from a given node, which may otherwise become excessively large in very long trains.

With the exception of the node installed in the locomotive, every node runs the same firmware, and only differs for a unique identification number (UID). The locomotive node, which we will also call master node, has an UID of zero. The UID is only used during the initialization phase. Afterwords, the nodes are addressed by means of the electromagnetic distance (ED) from the locomotive node, which is an integer number corresponding to the number of radio hops needed to reach the master node. The ED is assigned during the initialization phase; the master node has $\mathrm{ED}=0$.

\section{A. Network initialization}

The initialization (shown in the flow chart in the two leftmost sections of Fig. 2) is performed by means of a software token, which is generated by the master node and propagated towards the train tail. Only one node at a time can "hold" the token. At the startup of the network, the token is held by the master node. The other nodes are in the uninitialized mode and start transmitting announce packets as soon as their supercapacitors are fully charged (so that they will be able to operate continuously for several seconds) at pseudo random (a simple linear congruent generator is enough) intervals, in order to minimize collisions. The announce packets contain the sender UID. The token holder receives the announce packets and stores the sender UIDs and the received signal strength. After receiving a given number of packets or after a given amount of time, it selects the strongest (i.e. the one with the largest value of RSSI, the Received Signal Strength Indication), thereby probably belonging to the nearest node, and passes the token on to the corresponding node. Since the 


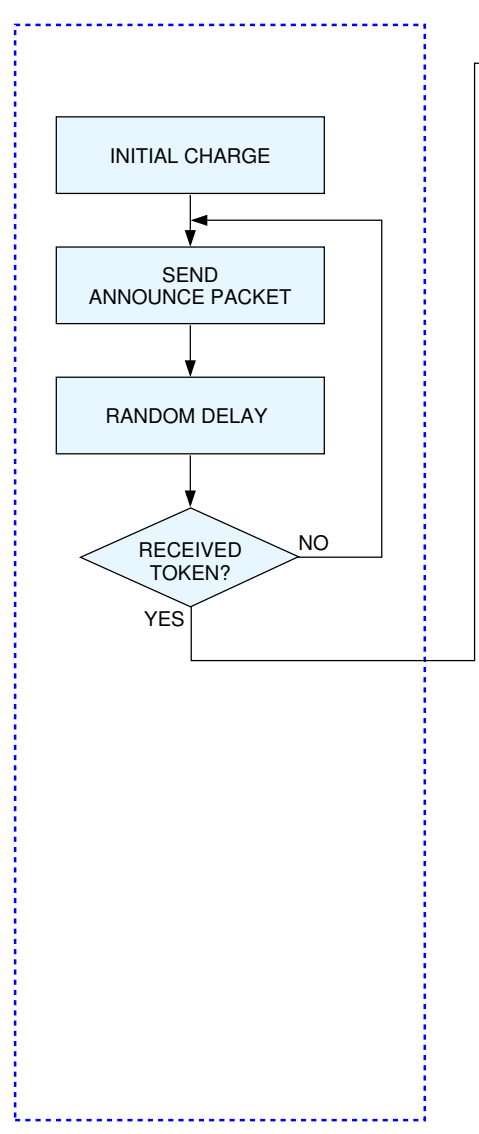

ANNOUNCE PHASE

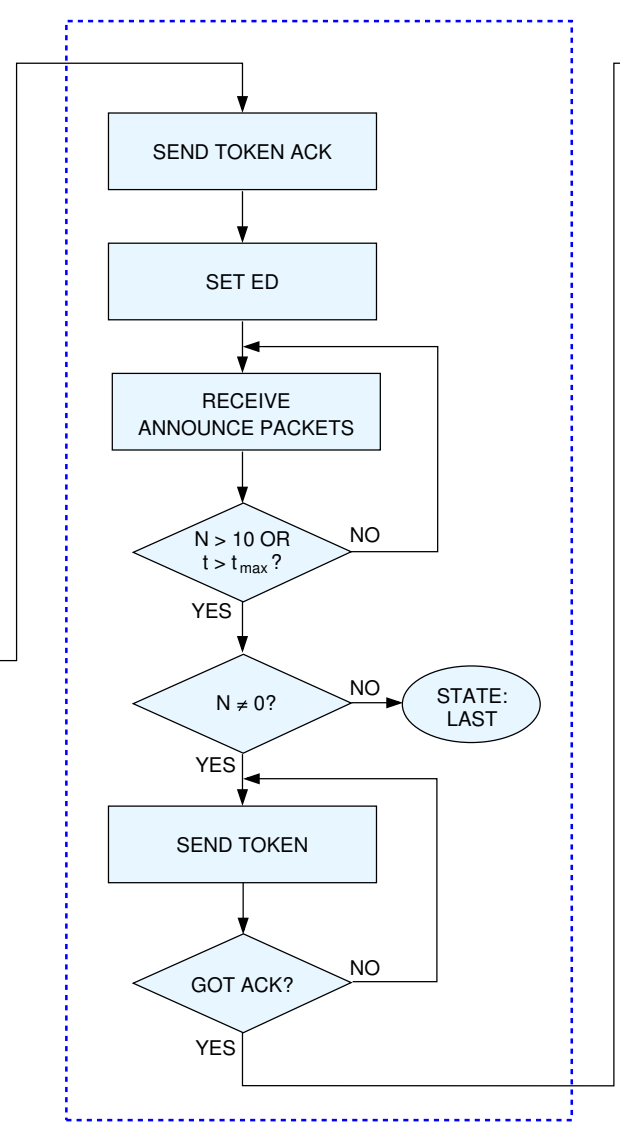

TOKEN PHASE

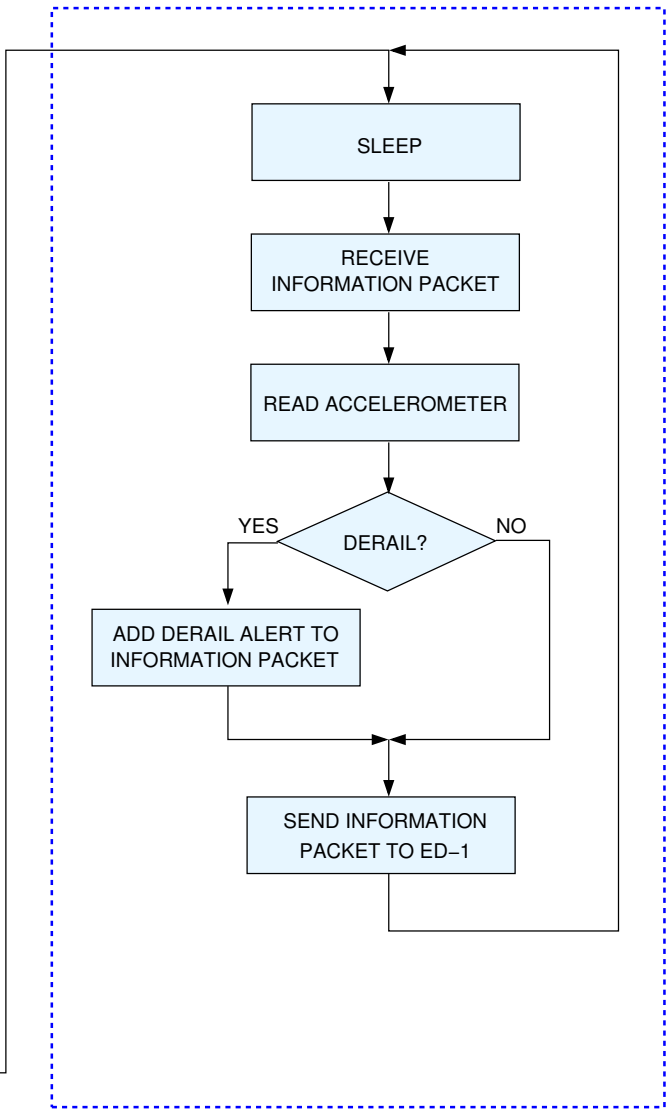

NORMAL OPERATION

Fig. 2. Flow chart of the initialization process and of normal operation

reliable transmission of the token is essential for the correct network initialization, the receiver node sends back an ACK (acknowledgment) packet to confirm token reception.

Every time the token is handed over to the next node, the $\mathrm{ED}$ is incremented and hence every node has a unique ED which reflects the order in which it received the token, which in turn is related to the distance from the locomotive. In order to prevent errors resulting from the fact that the signal arriving from the opposite side of the train may be significantly weaker and therefore lead to nodes being skipped, each node of the pair installed on the same car knows the UID of the other node, and forces its inclusion as nearest neighbor.

The last node holding the token cannot receive valid announce packets in the allotted time. Hence the token expires and the node assumes to be the last in the train (tail node).

In general, the larger the energy stored for the initialization phase, the longer such a phase can last. However, in order to have a larger amount of stored energy, the beginning of the initialization phase has to be delayed until such energy becomes available. Therefore the duration of the initialization phase should be a tradeoff between the acceptable delay after the train reaches a speed sufficient to charge the energy storage elements and the initialization time needed for the largest number of cars that are expected to be in the train.

\section{B. Network operation}

After the initialization phase the network begins the ordinary operation (see the rightmost section of Fig. 2). The tail node sends information packets with the derailment status information (just a single bit would be sufficient, at zero in normal conditions and at 1 when a derailment is taking place). The node with the next-to-last ED receives the packets, updates the status information and retransmits the packet. In this way the information is transported all the way to the master node. Every packet has a sequence number and hence the master node can detect missing packets. However, the frequency of the information packets ( 1 per second) is large enough to tolerate the sporadic loss of some packets.

\section{Handling of network failures}

Many network anomalies can lead to the presence of stray uninitialized nodes:

- A node misses the token during the initialization phase.

- The token expires at mid train leaving uninitalizated nodes at the tail.

- A node is restarted after a power shortage.

- A node does not receive packets for a while, times out and restarts. 
If properly configured nodes receive announce packets from stray nodes, they will add this knowledge to the information packets, to relay it the master node, which can trigger a global reconfiguration.

In order to trigger this reconfiguration, the master node sends a few reset packets. Every node receiving a reset packet resends it after a small random time and then resets. The reset packets are launched with a time-to-live counter which is decremented at every re-transmission. Hence they trigger a "reset storm" which extinguishes itself after some time, leaving every node in the uninitialized state. Afterwards the master node sends a new token.

The problem of nodes belonging to a nearby train being erroneously included in the initial network configuration is addressed in Ref. [17] on the basis of an evaluation of the speed of each node: nodes belonging to the same train will have the same speed. We do not intend to spend a relatively important computational effort for the evaluation of the speed (for example performing an FFT of the accelerometer output), since we believe that the inclusion of spurious nodes is not a real problem in practice. Since the configuration procedure takes place when the train has already reached a speed sufficient to charge the supercapacitors with the needed amount of energy, it is unlikely that another train whose nodes are also performing the configuration process will be on a nearby track. Furthermore, even if such an event occurred, the spurious nodes will be dropped a short time later, when the two trains will be at a distance such that radio communication between their nodes will not be possible any longer.

The packets consist of a byte which specifies the packet type and a variable length payload (the length depends on the packet type). For example, information packets are made up of: packet type, sender ED, 2 byte serial number, derailment status byte. They can be longer if additional functionalities are included, such as relaying temperature or humidity information, as discussed in the following. The maximum packet length is 16 bytes.

The serial number is important, because it allows the master node to determine whether any packet loss has taken place. A reconfiguration can be forced after a nonnegligible number of packets has been lost.

\section{IMPLEMENTATION AND DETECTION CRITERIA}

We have implemented a prototype network consisting of three nodes: the first corresponds to the one located at the end of the train, then there is one intermediate node, and, finally, a node corresponding to the master node to be located in the locomotive.

For most of the time, each node is in the ultra-low-power sleeping mode, waking up only once within each cycle, in order to read the accelerometer information and to transmit a message to be received and propagated by the other nodes. The current choice for the duration of the cycle has been $1 \mathrm{~s}$, this interval can be decreased at higher speeds, when plenty of energy is available from the harvesters and the timeliness of the derailment warning is more important.

There are two accelerometers (the LIS3DH and the ADXL362) on the test board for the purpose of evaluating both of them, in order to decide which one to install in the final node design: the LIS3DH has a definitely lower cost (1.63 Euro vs. 8.06 Euro), but a higher current in sleep mode $(0.5 \mu \mathrm{A}$ vs. $10 \mathrm{nA})$ and does not have a motion activated wake-up mode. This latter feature is however not particularly useful in our application, because acceleration measurements require only a small portion of the overall dissipated energy and operation of the network of sensors requires them to be performed with a given periodicity anyway. The fact that the energy used up by the accelerometer is (as will be shown in the following) negligible compared to that necessary for the CC1110 operation, makes the cheaper LIS3DH a preferred choice, also considering that it has a wider measurement range (up to $16 \mathrm{~g}$ ) which covers the activation threshold (11.5 g) used for mechanical derailment detectors, such as the EDT101 [2].

In the active phase of each node, timing is guaranteed by the $26 \mathrm{MHz}$ main clock crystal, but when the sleep-mode is entered, the time interval before returning to the active mode is measured based on the internal $R C$ oscillator of the microcontroller. This involves that such interval is affected by some fluctuations, which make it necessary to program each node in such a way that it will start listening in reception mode somewhat in advance, and therefore the overall power budget will be slightly increased. Thus, in order to warrant the correct reception of the packet from its nearest neighbor, each node will be switched on into receive mode, and it will remain active until a packet is actually received and then passed onto the next node (with the same information as in the received packet or with an emergency message if the local accelerometer has measured a large enough vertical acceleration that makes a derailment likely to have occurred). In order to reduce the wait time in reception mode and to avoid possible losses of synchronization due to the effect on the internal $R C$ oscillator of the large temperature variations possible in a freight train environment, the final boards will be equipped with a 32768 $\mathrm{Hz}$ crystal for the operation of the sleep-mode timer.

All of our nodes share the same hardware, for which detailed schematics can be found in Fig. 3. The different functionalities of the nodes are obtained by uploading different versions of the software. The main component is the CC1110 System-on-Chip from Texas Instruments, which includes a microcontroller with an ultra-low-power sleep mode and a transceiver operating in the $915 \mathrm{MHz}$ ISM band, used to communicate with the neighboring nodes. The accelerometer, which has an ultra-low-power sleep mode, too, is connected to the microcontroller via an SPI bus. As previously discussed, the prototype boards include two jumper-selectable accelerometers, for comparison purposes. The balanced output of the CC1110 is interfaced with the single ended antenna by means of a Johanson Technology balun (a passive-component integrated circuit), followed by a low-pass filter with a $2.13 \mathrm{GHz}$ cut-off frequency. In these prototype boards we have included also a serial RS232 port, connected to the microcontroller UART via a MAX3232 level translator. This port is used for debugging purposes and will not be present in the final versions of the boards. In the prototype boards the power supply to the MAX3232 can be disconnected, in order to be able to measure the power consumption due the components that are actually needed in normal operation. The CC1110 from Texas Instruments, which we have used for our current prototypes, has the possibility of entering an ultra-low-power 


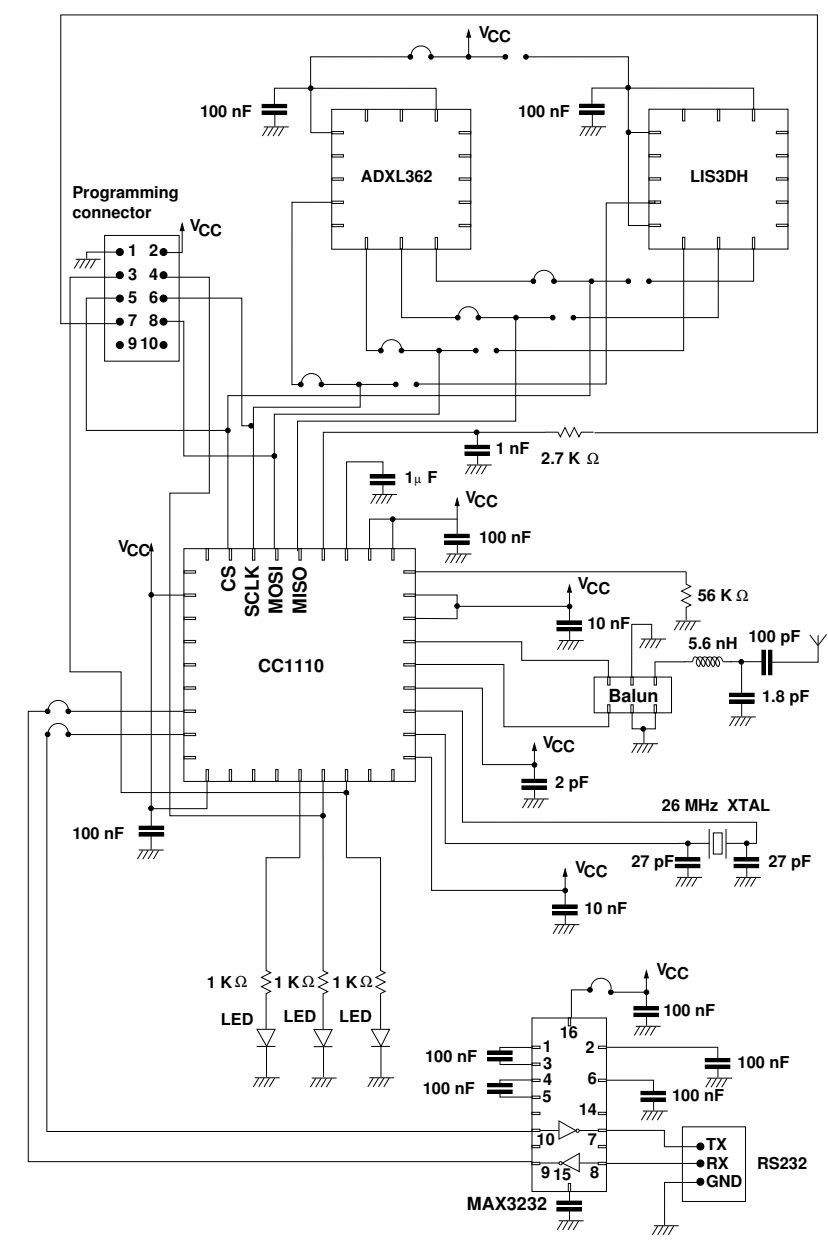

Fig. 3. Detailed schematic of a test node

sleep mode (requiring $0.5 \mu \mathrm{A}$ ) and to be woken up after a given time, evaluated on the basis of the cycles of a lowfrequency oscillator (which in the current prototypes is the internal $R C$ oscillator, while, as already stated, in the final production boards it will be based on a $32768 \mathrm{~Hz}$ crystal for improved timing accuracy). A picture of one of the prototype boards is shown in Fig. 4, with an indication of the main components.

The integrated transceiver can work with a channel attenuation of up to $120 \mathrm{~dB}$, since it has a sensitivity (at a data rate of $1.2 \mathrm{kBaud}$ ) of $-110 \mathrm{dBm}$ and a maximum output power of $10 \mathrm{dBm}$. At the higher bit rate of $250 \mathrm{kBaud}$ (the one we have been using in most of our tests, to keep the transmission and receiving phases as short as possible) the sensitivity is $-93 \mathrm{dBm}$, therefore a total channel attenuation of $103 \mathrm{~dB}$ is allowed.

We also plan to experiment with the CC430 integrated microcontroller-transceiver, which is interesting for the presence of a low-power real time clock, which could simplify the synchronization among the nodes.

As far as detection criteria are concerned, Kato and Tenada present three main concepts in Ref. [6]: comparison of the absolute amplitude of the vertical acceleration with a threshold, comparison between the integral of the modulus of the vibrations within a given time interval and that in a previous

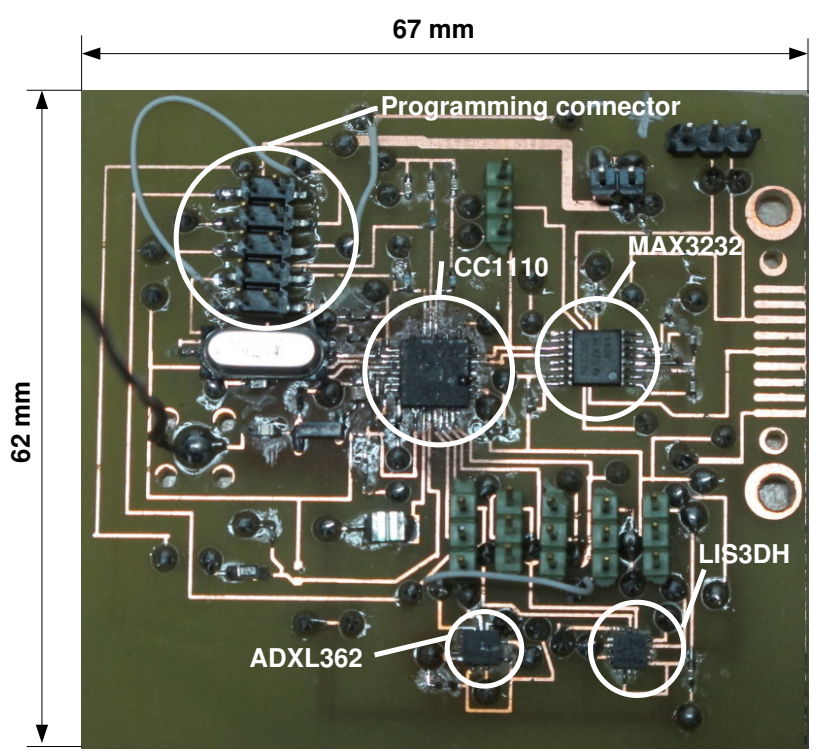

Fig. 4. Photograph of a prototype node used for testing

interval, comparison of the double integral of the acceleration over an interval of $200 \mathrm{~ms}$ with the $90 \mathrm{~mm}$ of the height of the rail profile. In terms of energy saving the first criterion is definitely to be preferred, because it requires an observation window, and therefore an active operation window, that can be very short. In order to reduce false positives, some form of the second criterion could be adopted, to be used as a confirmation if on the basis of the first a derailment condition is detected, while the third criterion is not suitable for our system because it would require a continuous observation.

We would like to point out that the sensor network for derailment detection could be used also for other purposes, since there is an interest by freight train operators to monitor some environmental parameters in the cars, such as temperature or humidity. These quantities could be measured with sensors built in the cars or in the containers loaded on the cars, and then transferred to the derailment detection nodes, which could periodically relay them to the main node on the locomotive, as long there is no derailment condition active. Another potentially very interesting functionality could be implemented if the system became widely accepted and present on the majority of existing rolling stock, with all locomotives equipped with a master node. In such a case it would be possible to track the position of all freight cars, because each node can be tagged with a unique identifier that is associated with the car itself. Every time a car is coupled to a train that at some time moves, it would be included in the consist listing stored in the master node of the locomotive, which can send it, together with the position of the train, to a central location via GSM-R or other long-distance radio link.

\section{EXPERIMENTAL RESULTS}

We have focused on the experimental evaluation of the average power consumption by each node, which is hard to determine with precision directly from the data sheet, due to the relatively complex sequence of states through which the radio part of the $\mathrm{CC} 1110$ will have to cycle in order to perform a wake from the ultra-low-power sleep condition, 

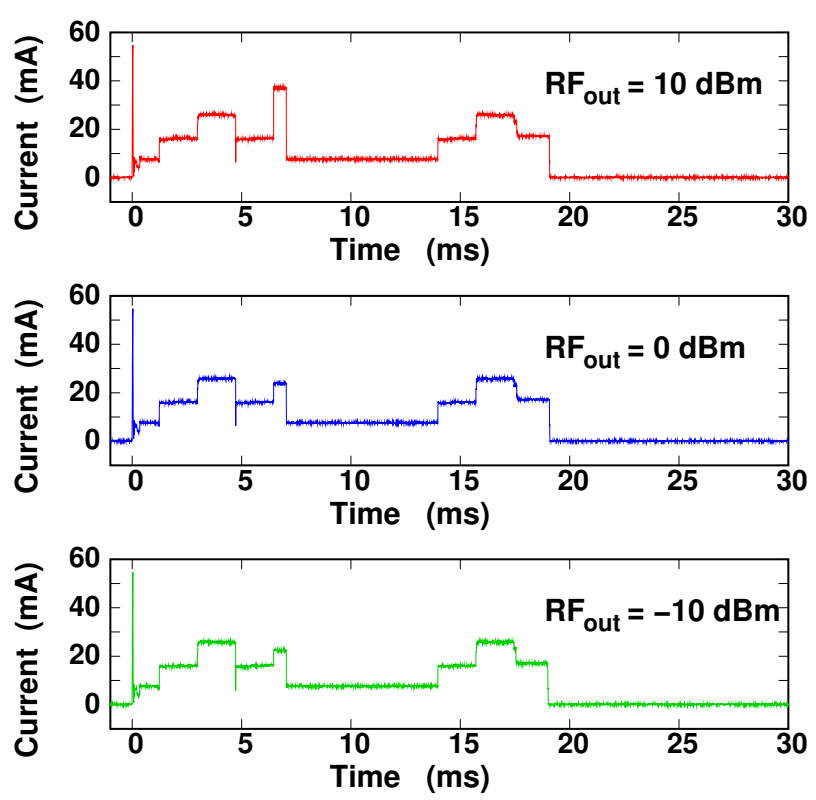

Fig. 5. Comparison of the current drawn during a test sequence for an output power of $10 \mathrm{dBm}$ (top panel), $0 \mathrm{dBm}$ (center panel), -10 dBm (bottom panel)

receive a packet from the previous node, forward it to the next node and listen to the possible transmission from a local environmental (temperature, humidity, etc.) sensor (for whose forwarding there is no particular urgency constraint, so that it can be delayed to the following packet transmission). The top curve of Fig. 5 represents the current supplied to the prototype node (at a voltage of $3 \mathrm{~V}$ ) when it is woken up from deep sleep (at $t=0$ ) and then completes a transmission burst at a power level of $10 \mathrm{dBm}$, preceded by a receive interval (to get information from the previous node) and followed by a second receive interval (to acquire the information from other sources on the car, if available). There are some minimum times that have to be spent for the stabilization of the internal VCO (Voltage Controlled Oscillator) and overall settling of the $\mathrm{RF}$ section. It is to be noted that, as far as the data from the temperature, humidity, or other sensors are concerned, during each complete cycle (of the order of $1 \mathrm{~s}$ in a typical train) only the information relative to one single car is forwarded to the locomotive, otherwise an excessive number of bytes would be needed, if information about all cars were to be forwarded at the same time. This is not a problem, because the measured environmental quantities vary very slowly or, in the case of payload information, do not vary at all.

At $t=0$ we observe a short burst, whose detailed shape is shown in the enlargement of Fig. 6, and which is most likely the result of the activation of the digital core voltage regulator and charging of the associated external $1 \mu \mathrm{F}$ filter capacitor. Then, after switching through the intermediate states, a receive interval begins at about $3 \mathrm{~ms}$ and lasts for about $2 \mathrm{~ms}$. At about $6.5 \mathrm{~ms}$ the transmission burst starts, lasting for about $500 \mu \mathrm{s}$. With the current software we transmit the 11 bytes needed by the protocol ( 6 preamble bytes, 2 synchronization bytes, one byte containing the length of the packet, one byte for the source address and one byte for the destination address) followed by 3 bytes of information (in the final implementation there will also be 2 additional CRC bytes). With the output power set at

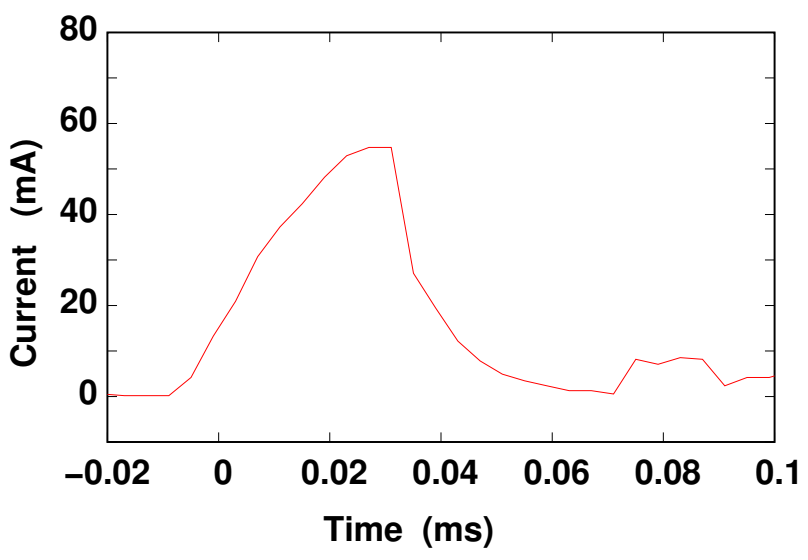

Fig. 6. Enlargement of the current pulse appearing immediately after the wake up from deep sleep

the maximum value of $10 \mathrm{dBm}$, the current draw is of about $37 \mathrm{~mA}$. Current measurements have been performed supplying to the input of a Tektronix TDS 784 oscilloscope the voltage drop across a $2.7 \Omega$ resistor in series with the power supply of the prototype board.

In the middle and bottom panels of Fig. 5 we report the time dependence of the current drawn for an output power of $0 \mathrm{dBm}$ and of $-10 \mathrm{dBm}$, respectively. It is possible to notice that the current needed during the transmission burst decreases from $37 \mathrm{~mA}$ to $24 \mathrm{~mA}$ if the power is lowered to $0 \mathrm{dBm}$, but further lowering to $-10 \mathrm{dBm}$ leads to a very small change of the current, down to about $22.5 \mathrm{~mA}$. Therefore, also considering the short duration of the transmission burst (compared to the overall active interval) we conclude that a tight management of the output power is not essential from the point of view of the optimization of the energy balance.

During the receive operation the current drawn is about $27 \mathrm{~mA}$, which corresponds (at $3 \mathrm{~V}$ ) to a power of $81 \mathrm{~mW}$. This represents the most critical aspect of the initialization phase, during which, in order to receive the token, the nodes must be in receive mode most of the time. Since the initialization time is about $30 \mathrm{~ms}$ per node, assuming 200 nodes (2 nodes per car and 100 cars), the total initialization time is about $6 \mathrm{~s}$. Hence the total energy that must be collected before starting the configuration phase is $486 \mathrm{~mJ}(81 \mathrm{~mW}$ for $6 \mathrm{~s})$. Since, from Ref. [8], a power of about $80 \mathrm{~mW}$ can be obtained from an electromagnetic scavenger at the speed of $80 \mathrm{~km} / \mathrm{h}$, the power that can be expected at a speed of $20 \mathrm{~km} / \mathrm{h}$ is about $5 \mathrm{~mW}$ (the amplitude of the vertical acceleration can be assumed to be proportional to the square of the train speed and the power scavenged has an approximate linear dependence on the vertical acceleration). This implies that at $20 \mathrm{~km} / \mathrm{h}$ a time of about $97 \mathrm{~s}$ is needed to collect the energy required to perform the network initialization.

In order to evaluate the overall energy used during an active interval, we have multiplied the data in the top curve of Fig. 5 by the supplied voltage of $3 \mathrm{~V}$ and then integrated over time, obtaining a value of $878.5 \mu \mathrm{J}$. If we consider that this cycle, of the approximate duration of $20 \mathrm{~ms}$, has to be performed once within an overall cycle, for example, of $2 \mathrm{~s}$, the average power needed will be of $439.25 \mu \mathrm{W}$. We should also add the contribution from the power used up during the rest of the 
time in the deep-sleep mode, but it is negligible, since the current needed in this condition is below a microampere. The same can be said for the power needed by the accelerometer, which, at most, requires a few microamperes during the active operation and for the rest of the time needs only a fraction of a microampere.

As far as the energy required by the CC1110 for the acquisition and processing of the accelerometer data is concerned, we can estimate 5 readings and evaluations per overall interval (2 s), each of which takes about $2 \mathrm{~ms}$, for a total of $10 \mathrm{~ms}$.

Considering that the processor requires $5 \mathrm{~mA}$ in active mode, this leads to an energy consumption of $150 \mu \mathrm{J}$ per overall interval. Therefore a reasonable estimate of the average power required can be obtained summing up the two leading energy terms (from the communication protocol and from the accelerometer data reading and processing) and dividing by the duration of the outer cycle $(2 \mathrm{~s})$. This yields a value of $514.25 \mu \mathrm{W}$, which can be certainly supplied by induction scavengers, but likely also by cheaper piezoelectric scavengers. Since the cost of the electronics is of the order of about 20 Euro, the main contribution to the overall cost is from the scavenger, therefore optimization of the energy consumption can lead to significant cost reductions.

We have then focused on the implementation of a simplified prototype network, based on the concepts that we have previously discussed. On this network we have successfully tested both the configuration algorithm and the normal operation mode. In normal operation we have, as previously stated, one tail node sending a burst of data approximately every second; this burst is received by the intermediate node, which, after a very short time, forwards the information towards the head node. In Fig. 8 we report the evolution of the current: the top (green) curve corresponds to the current supplying the tail node, which performs only a transmit operation; the middle (blue) curve represents the current used by the middle node, which is switched on in receive mode after a sleep period, and then retransmits the information that has received; finally the bottom (red) curve is relative to the current flowing into the head node, which receives the information sent by the middle node. Aside from the bottom one, the curves are vertically shifted for clarity of presentation: the middle one by $80 \mathrm{~mA}$ and the top one by 160 ma. Except for the free-running tail node, the other nodes are synchronized by the end of the transmission that they receive: as soon as the received packet is over, the node goes into sleep mode for a time close to $1 \mathrm{~s}$, evaluated on the basis of the internal $R C$ oscillator. Due to the limited stability of such an oscillator, the receiver must be turned back on again a bit earlier, with respect to the beginning of the new packet to be received, in order to have some margin to compensate for timing errors. We have made an estimate of the standard deviation of the sleep time interval by acquiring the supply current of the tail node for 50 cycles (see Fig. 7) with a resolution of $20 \mu$ s: we have then performed a correlation integral of the whole time record with that for a single cycle, in order to determine the position of each cycle. A more precise estimate has then been obtained looking for the sharp transition occurring at the end of the transmission phase. In this way we have estimated a standard deviation of about $0.24 \mathrm{~ms}$, which allows stable operation of the network (our test network has demonstrated stable operation for a whole day).

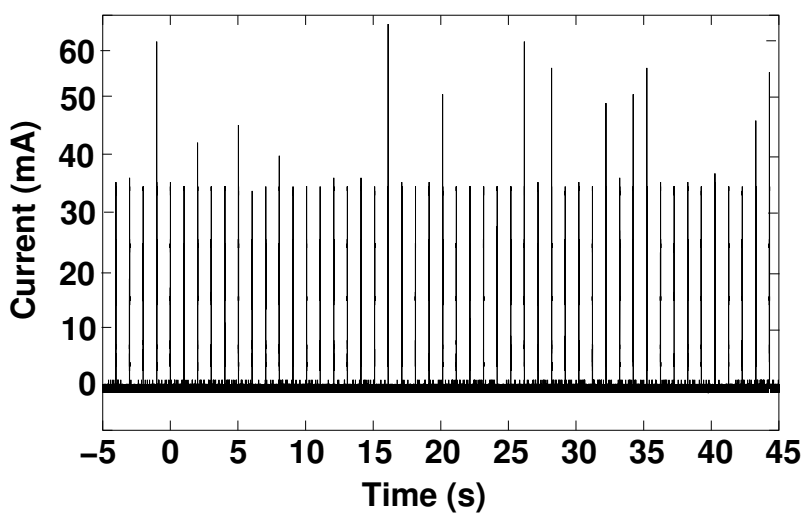

Fig. 7. Record of the current drawn by the tail node, over 50 seconds, for the evaluation of the standard deviation of the sleep intervals

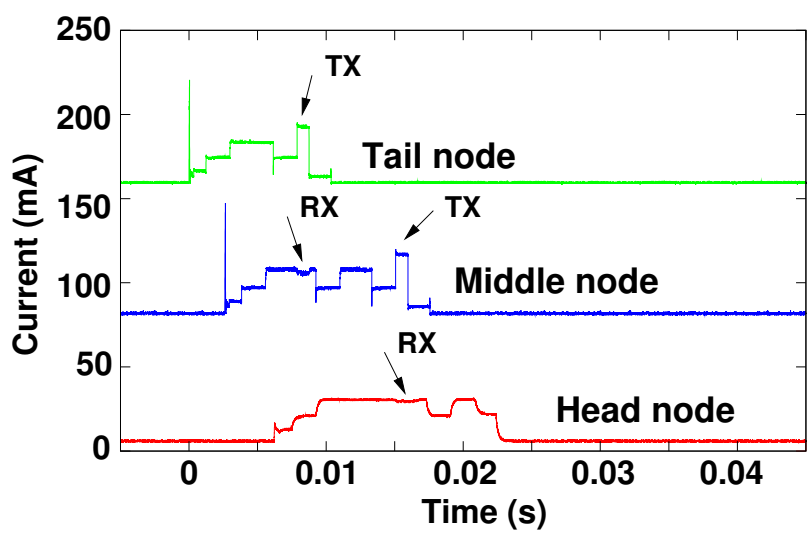

Fig. 8. Currents drawn by the three nodes forming a test network: the top, green, curve is relative to the tail node; the blue curve represents the current for the middle node; and the red curve that for the head node. For clarity of presentation the blue curve is shifted up by $80 \mathrm{~mA}$ and the green curve by $160 \mathrm{~mA}$

In order to verify the way in which communication among the nodes can be affected by the conductive surfaces of an actual train, we have performed a test in the operational mode on a three-car train at the Osmannoro facility of Italcertifer S.p.A.. The consist was made up of a freight car for gravel transportation (15.45 m long) placed between two UIC-X passenger cars (each $26.4 \mathrm{~m}$ long, therefore much longer than most freight cars). We experimented with different positions for the three nodes, which were equipped with basic $\lambda / 4$ antennas (always in a vertical position), and were operating at the maximum transmitting power of $10 \mathrm{dBm}$. Since the train was stationary, power to each node was supplied by two AA $1.5 \mathrm{~V}$ cells. Some of the tested configurations are sketched in Fig. 9. In all configurations we were able to obtain a stable operation of the three-node network, with received signals that ranged from the $-60 \mathrm{dBm}$ of configuration a) to the about $-70 \mathrm{dBm}$ for the most critical link, i.e. that between the middle and right node in configuration $b$ ). The largest attenuation was observed when nodes are on opposite sides of the car, as expected. We repeated the tests, without observing significant differences, with the nodes attached to the side of the car at various heights, from about $1 \mathrm{~m}$ to $1.50 \mathrm{~m}$ above the rail or also placed on the truck frames. We have also observed that communication was still possible 
a)

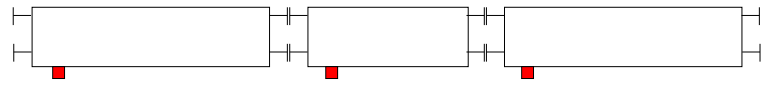

b)

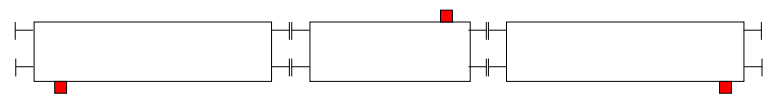

c)

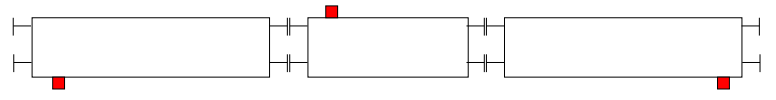

d)

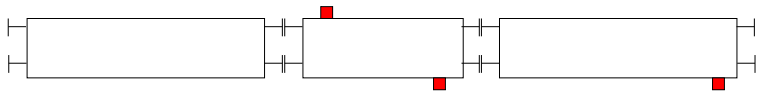

e)

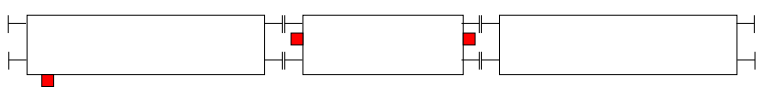

Fig. 9. Configurations of the nodes in the tests performed on actual rail cars

between two nodes placed at the ends of the complete threecar consist. In all configurations we tested the transmission of 128 packets, all of which were received correctly. The test thus demonstrated the communication between nodes in an actual train environment.

Therefore, considering that further improvement can be achieved using antennas with better directionality (and therefore higher gain) and assuming to install two nodes on each car in the positions corresponding to those for the middle car of configuration d), reliable communication should be possible also in the presence of two consecutive cars without nodes or with non functional nodes.

In order to evaluate the effect of electromagnetic interferences from the locomotive or from other trains and unexpected attenuation due to large metallic surfaces, it is therefore important to envisage a series of measurements to be performed on freight cars in regular operation. To this purpose we are designing a system (which can be temporarily installed on one or more freight cars) capable of acquiring the vibration spectrum, evaluating the performance of one or two different scavengers, testing communication with a couple of nodes placed on adjacent cars. Such a system should be attached to the body of the car and have batteries to guarantee operation in all conditions. It should also have a slave unit at the other end of the car, transmitting packets at variable power levels, in order to verify the reliability of the communication between nodes in the different electromagnetic environments that a train will be going through. This will also provide us with information about how the RSSI (which we use during the configuration phase) may be affected by external interferences.

Furthermore, once a new version of the node (designed on the basis of the results of the above-mentioned tests) will be available, we plan to investigate the performance of the synchronization algorithm with an increasing number of nodes, in order to finally validate it for operation with trains with over a hundred cars.

\section{CONCLUSion}

We have devised a network of wireless sensors for the detection of the derailment of cars in freight trains, which can serve also additional purposes, such as the collection of temperature and humidity data, or of payload information. On the basis of the knowledge of typical freight operation, we have focused on the requirements of low cost and of low maintenance, and discussed the resulting design choices also in relationship with the existing literature. The specific design and the implementation of a prototype wireless sensor network have been undertaken, and experimental results have been discussed, pointing out the most critical issues, as well as a strategy for further development. In particular, an average power consumption of less than $0.5 \mathrm{~mW}$ per node has been achieved, with an active time of around $20 \mathrm{~ms}$ per cycle and with reliable communication over distances above $65 \mathrm{~m}$ in the realistic context of a train. A potentially critical issue is represented by the amount of energy that can be obtained from the energy scavenger: this in turn depends on the amplitude of vibrations at the different train speeds and will determine the time needed to collect enough energy to sustain a successful initialization phase. We have defined a strategy for further research, useful to better define these issues, based on measurements performed on a freight car operated in an actual railway environment.

\section{ACKNOWLEDGMENT}

We acknowledge financial support from the project CTN01_00176_166195 - MIUR "ITS (Sistema di Trasporto Intelligente) Italia 2020" CUP B48F13000640008. We would also like to thank Italcertifer S.p.A. for giving us the possibility to test our nodes on real rail vehicles on their premises.

\section{REFERENCES}

[1] European Railway Agency. (2009). Impact assessment on the use of derailment detection devices in the EU railway system, ERA/REP/03-2009/SAF. Available: http://www.era.europa.eu/DocumentRegister/Pages/final-report-on-derailment-detection-device.aspx [February 2, 2016].

[2] Knorr Bremse. EDT101 Derailment Detection System. Available: http://www.knorrbremse.de/en/railvehicles/products/trainsafety/edt101.jsp [February 2, 2016].

[3] RID Committee of Experts. Recommendation requiring the use of Derailment Detection Devices, Text provisionally adopted during the 44th session of the RID 2007 Committee of Experts meeting in Zagreb.

[4] A. V. Vostroukhov, A. V. Metrikine, A. C. W. M. Vrouwenvelder, V. I. Merkulov, V. N. Misevich, G. A. Utkin, "Remote detection of derailment of a wagon of a freight train: theory and experiment," Arch. of Appl. Mech., vol. 73, p. 75, 2003.

[5] M. Macucci, S. Di Pascoli, P. Marconcini, B. Tellini, "Wireless sensor network for derailment detection in freight trains powered from vibrations," in 2015 IEEE International Workshop on Measurements \& Networking $(M \& N)$, Coimbra, Portugal, 2015, pp. 36-41. doi: 10.1109/IWMN.2015.7322970

[6] M. Kato, K. Terada, "Development of and Evolution Type Train Protector System to Prevent Secondary Accidents," in International Rail Safety Conference, Dublin, Ireland, 2006.

[7] Energy Harvester PMG37 by Perpetuum Ltd.. Available: http://www.perpetuum.com [February 2, 2016].

[8] G. De Pasquale, A. Somà, N. Zampieri, "Design, Simulation, and Testing of Energy Harvesters with Magnetic Suspensions for the Generation of Electricity from Freight Train Vibrations," Journal of Computational and Nonlinear Dynamics, vol. 7, p. 041011, 2012.

[9] A. Rahimi, Ö. Zorlu, A. Muhtaroğlu, H. Külah, "Fully Self-Powered Electromagnetic Energy Harvesting System with Highly Efficient Dual Rail Output," IEEE Sensors Journal, vol. 12, p. 2287, 2012. 
[10] C. Ung, S. D. Moss, L. A. Vandewater, S. G. Galea, W. K. Chin, G. Crew, "Energy Harvesting from Heavy Haul Railcar Vibrations," in 2013 IEEE Eighth International Conference on Intelligent Sensors, Sensor Networks and Information Processing, Melbourne, Australia, 2013, p. 95. doi: 10.1109/ISSNIP.2013.6529771

[11] M. G. Tehrani, G. Gatti, M. J. Brennan, D. J. Thompson, "Energy Harvesting from Train Vibrations," in 11th International Conference on Vibration Problems, Lisbon, Portugal, 2013.

[12] M. Lallart, S. Pruvost, D. Guyomar, "Electrostatic Energy Harvesting Enhancement Using Variable Equivalent Permittivity," Physics Lett. A, vol. 375, p. 3921, 2011.

[13] S. Roundy, P. K. Wright, J. M. Rabaey, Energy Scavenging for Wireless Sensor Networks with Special Focus on Vibrations. Norwell, MA: Kluwer Academic Publishers, 2004.

[14] H. Vocca, I. Neri, F. Travasso, L. Gammaitoni, "Kinetic Energy Harvesting with bistable oscillators," Applied Energy, vol. 97, p. 771, 2012.

[15] H. Külah, K. Najafi, "Energy Scavenging from Low-Frequency Vibrations by Using Frequency Up-Conversion for Wireless Sensor Applications," IEEE Sensors Journal, vol. 8, p. 261, 2008.

[16] G. De Pasquale, A. Somà, and F. Fraccarolo, "Piezoelectric energy harvesting for autonomous sensor network on safety-improved railway vehicles," Proceedings of the Institution of Mechanical Engineers, Part C: Journal of Mechanical Engineering Science, vol. 226, p. 1107, 2012. doi: 10.1177/0954406211418158

[17] G. A. Barone, J. O. Breitfeller, D. A. Jacobs, C. W. Nyquist, R. E. Rose Jr., "Self-assembling wireless network, vehicle communication system, railroad wheel and bearing monitoring systems and methods therefor," U.S. patent US7705743 B2, April 27, 2010.

[18] E. Sardini, M. Serpelloni, "Self-Powered Wireless Sensor for Air Temperature and Velocity Measurements With Energy Harvesting Capability," IEEE Trans. Instrum. Meas., vol. 60, p. 1838, 2011.

[19] H.-C. Lee, Y.-C. Chang, and Y.-S. Huang, "A Reliable Wireless Sensor System for Monitoring Mechanical Wear-Out of Parts," IEEE Trans. Instrum. Meas., vol. 63, p. 2488, 2014.

[20] A. Hać, Wireless Sensor Network Designs. Chichester, England: John Wiley \& Sons, 2003.

[21] C. K. Harnett, M. T. Schueler, N. R. Blumenthal, K. L. Hopf, J. F. Fox, S. Pulugurtha, "Calibration and Field Deployment of Low-Cost Fluid Flow-Rate Sensor Using a Wireless Network," IEEE Trans. Instrum. Meas., vol. 60, p. 633, 2011.

[22] O. Rorato, S. Bertoldo, C. Lucianaz, M. Allegretti, G. Perona, "A multipurpose node for low cost wireless sensor network," in 2012 IEEE-APS Topical Conference on Antennas and Propagation in Wireless Communications (APWC), Cape Town, South Africa, 2012, p. 247. doi: 10.1109/APWC.2012.6324911

[23] M. Magno, T. Polonelli, L. Benini, E. Popovici, "A Low Cost, Highly Scalable Wireless Sensor Network Solution to Achieve Smart LED Light Control for Green Buildings," IEEE Sensors Journal, vol. 15, p. 2963, 2015.

[24] A. Berger, M. Pichler, J. Klinglmayr, A. Pötsch, A. Springer, "LowComplex Synchronization Algorithms for Embedded Wireless Sensor Networks," IEEE Trans. Instrum. Meas., vol. 64, p. 1032, 2015. 